\title{
Open removal as a first-line treatment of magnetic intravesical foreign bodies
}

\author{
Max A. Levine, BSC (Kin); Howard Evans, MD, FRCSC
}

Division of Urology, University of Alberta, Edmonton, $A B$

Cite as: Can Urol Assoc J 2013;7(1):E25-E28. hitp://dx.doi.org/10.5489/cuaj.12043

\section{Abstract}

Intravesical foreign bodies are an uncommon, but significant, cause of urologic consultation. We present 3 patients who all inserted magnetic beads per urethra into the urinary bladder, which subsequently became retained. Endoscopic attempts were unsuccessfully tried in the first 2 cases, necessitating open cystotomy to remove the beads. The third went straight to open removal. Given the failure of minimally invasive techniques, we believe that open removal should be the first-line treatment for these types of foreign bodies.

\section{Introduction}

This is a case series describing 3 patients who presented independently to our group with unique foreign bodies in the bladder over the course of 9 months. These patients all had round magnetic beads (earth magnets) present in the urinary bladder. We describe these cases and the ensuing treatment. Based on our experience, we recommend open surgical approach for intravesical foreign body removal as a first approach when objects possess certain characteristics common to the cases presented.

\section{Patient 1}

Our first patient, a 42-year-old male, presented to the emergency department with concerns of foreign bodies in his urethra. On history, he admitted to finding magnetic beads hanging from the distal end of his urethra after a night of significant alcohol consumption. The beads were supposedly inserted while he was unconscious, and when he attempted to remove them manually, he felt as if he only removed the most distal beads, leaving some more proximally in the urethra.

A pelvic x-ray ordered by the emergency doctor revealed a radioopaque intravesical foreign body (Fig. 1a). He was sent to cystoscopy the next day for attempted extraction.
In cystoscopy, the urethra was noted to be relatively tight and scarred, suggesting a history of chronic urethral manipulation. The round magnetic beads were easily visualized in the bladder as a clump, but were irretrievable via endoscopic techniques. Both flexible and rigid cystoscopic attempts were ineffective, including the use of rat tooth graspers and baskets. The magnetic attraction between the beads made it impossible to remove them piecemeal or en bloc per urethra.

Cystoscopy was aborted and the patient was booked for open cystotomy. A lower midline incision was used to expose the bladder. An incision into the bladder allowed for manual exploration of the foreign bodies in situ, and extraction using Kelly clamps. The patient was discharged the next day with no complications.

Follow-up cystogram at 1 week after the operation revealed no leakage from the bladder, and no significant residual contrast. The catheter was removed and the patient's course thereafter was unremarkable.

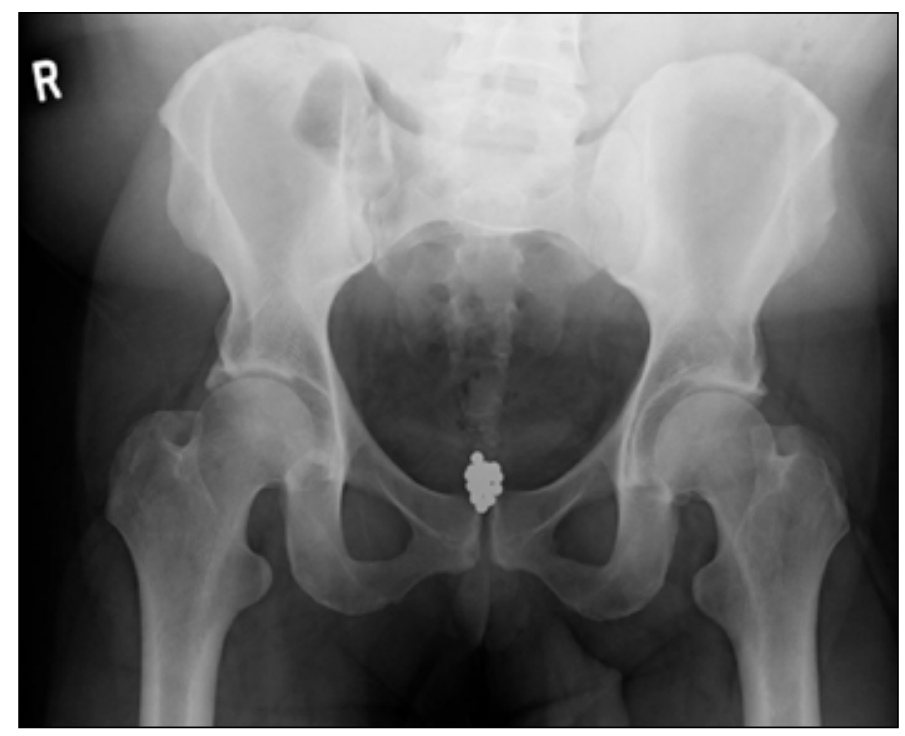

Fig. 1a. Patient 1, supine pelvic $x$-ray. 


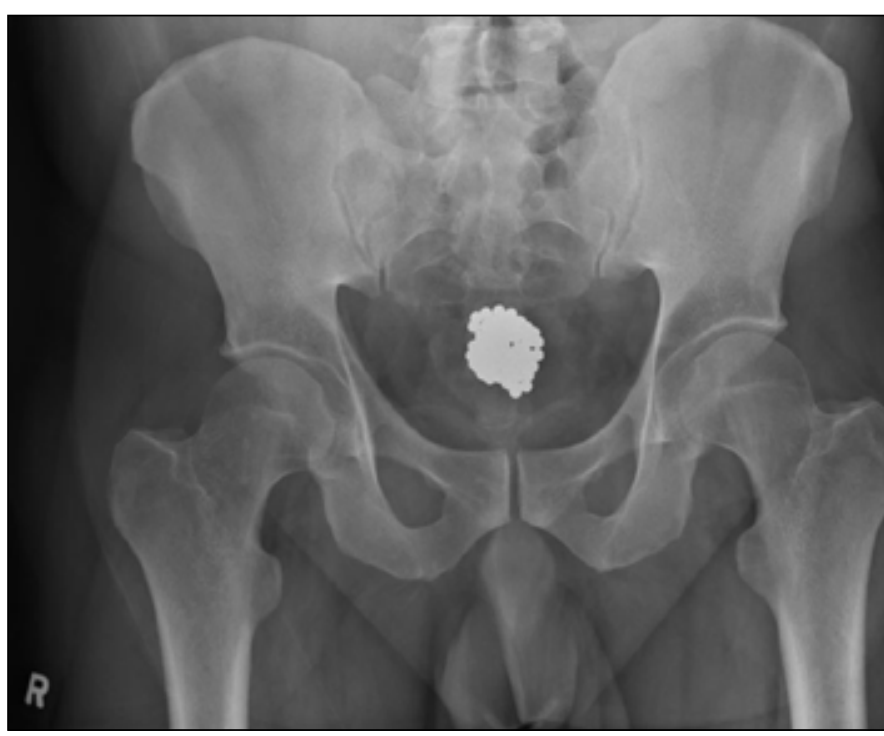

Fig. 1b. Patient 2, supine pelvic x-ray.

\section{Patient 2}

Our second patient, a 43-year-old male, presented to hospital requesting to see a surgeon about retained magnetic beads self-inserted per urethra. He claimed to have increasing lower urinary tract symptoms (LUTS) secondary to the foreign bodies (Fig. 1b).

Urologic consultation prompted a decision to proceed to the operating room that same evening to attempt a cystoscopic removal with possible open extraction. Once inside the bladder cystoscopically, the surgeons saw a clump of round magnetic beads. Graspers and baskets were tried in an attempt to extract the beads. The attractive force of the magnetic beads was too great to be overcome by these methods.

Cystoscopy was discontinued and the patient was redraped for open cystotomy. A small suprapubic incision allowed for identification of the bladder. The bladder was subsequently incised (about $2 \mathrm{~cm}$ ), which allowed for the removal of all the magnetic beads intact. An indwelling Foley catheter was removed the next day, and the patient was discharged without complications.

\section{Patient 3}

A third patient, a 30-year-old male, came to the emergency department after having recently self-inserted nearly 50 round magnetic beads into his urethra which he was unable retrieve; as a result, he was in urinary retention. He admitted to having inserted objects per urethra before, but this was the first incident of retention. Because of the recent experience with endoscopic failure described in the previous 2 cases, this patient was taken directly to the operating room for open removal (Fig. 1c).

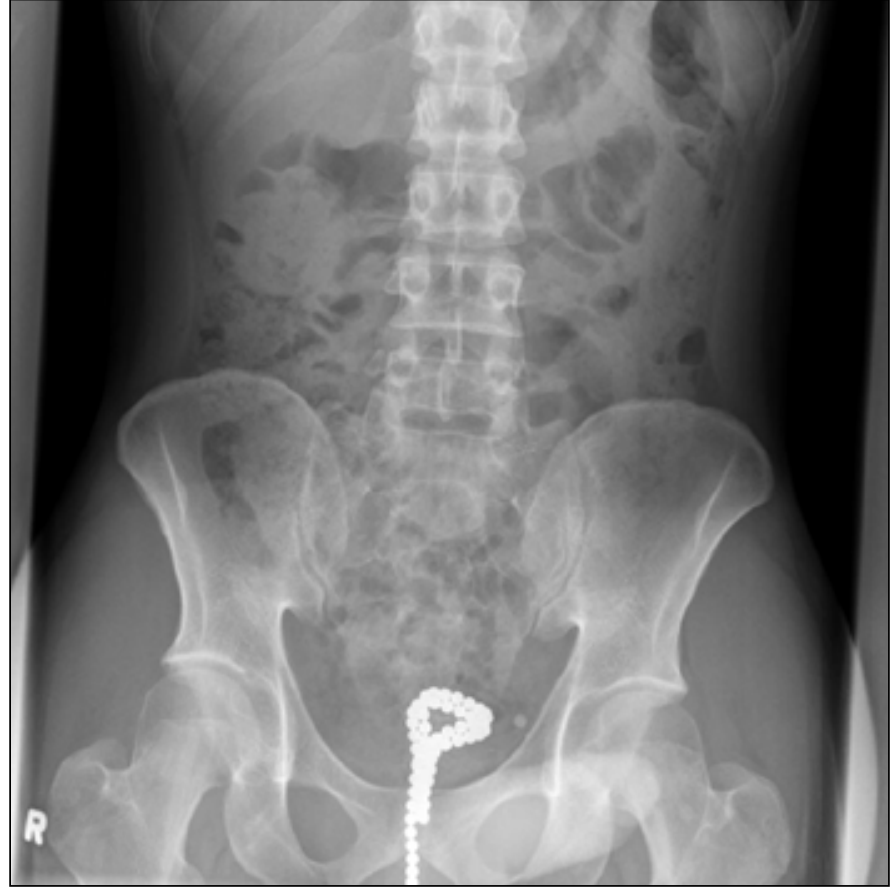

Fig. 1c. Patient 3, supine abdominal $x$-ray.

A lower midline abdominal incision was extended to the space of Retzius, where a vertical incision into the bladder was made and the round magnetic beads were extracted using metal-ring forceps. Intra-operative flexible cystoscopy was done to confirm no foreign bodies were left in the urethra. There were no residual beads found.

The patient was discharged the next day with a Foley catheter left in place. He returned to emergency room the following day with a complaint of hematuria per Foley. Laboratory and physical exam showed he was hemodynamically stable; the hematuria was part of an acceptable postoperative course. He came to the clinic the next day for follow-up, with no further issue.

\section{Discussion}

Foreign bodies in the bladder must always exist as a differential diagnosis for LUTS. ${ }^{1}$ While not necessarily the most common cause of LUTS, foreign bodies need to be considered to manage patients appropriately.

Most foreign bodies in the bladder are amenable to removal transurethrally. Therefore, endoscopic removal suffices for extraction of most foreign bodies, and should be considered the first-line approach. ${ }^{1,2}$ Self-inflicted foreign bodies vary in size, shape and malleability. Documented objects described in urinary bladders have included batteries, wires, hair pins and screws. ${ }^{1-4}$ A singular approach for all intravesical objects is inappropriate. Moon and colleagues found that the exact strategy for foreign body removal from the bladder is dictated by the size, location and mobility of the object. ${ }^{2}$ 


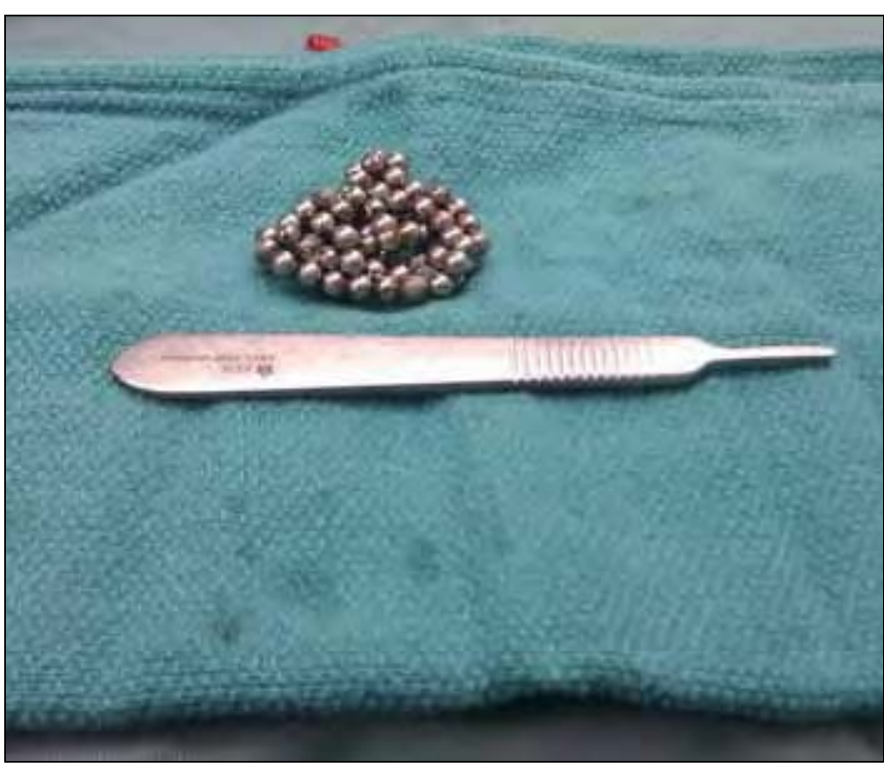

Fig. 2a. Clumped magnetic balls post-extraction, with scalpel handle for perspective.

In our cases, endoscopic removal was ineffective. The earth magnets we found were beads that are found in toy stores (Fig. 2a, Fig. 2b). These magnets have an attractive force that creates a bunching of the magnets in the spacious bladder after emerging from the urethral conduit. The magnetic attractive force is sufficiently strong that once they clump, they cannot be separated with endoscopic techniques. Our first 2 cases demonstrated this. Similar to the clumping of magnets, Eradi and Shenoy detail a case of an insulated electrical wire inserted into the bladder transurethrally that spontaneously knotted once inside. ${ }^{3}$ Through an endoscopic exam, the examiners decided that open surgical extraction was required.

The ultimate goal in the extraction of foreign bodies from the bladder is to do so with minimal harm to the bladder and urethra. ${ }^{2}$ The recommendation for minimally invasive removal as a first-line treatment is therefore justified in most cases. However, some objects may possess attributes that are predictive of difficult cystoscopic extraction. Size and mobility may be the first indications. Even though objects may be capable of traversing the urethra in a retrograde fashion, antegrade retrieval may not be possible. ${ }^{5}$ While no absolute diameter or length of object has been described as prohibitive of endoscopic removal, common sense should prevail, considering the relatively high resistance anatomic path the object must take upon extraction.

An important consideration should be the mechanics of the inserted foreign body. In our cases, the string of magnetic beads that were inserted per urethra in a linear fashion easily clumped once inside the bladder. The dynamic properties of these magnets necessitated open removal.

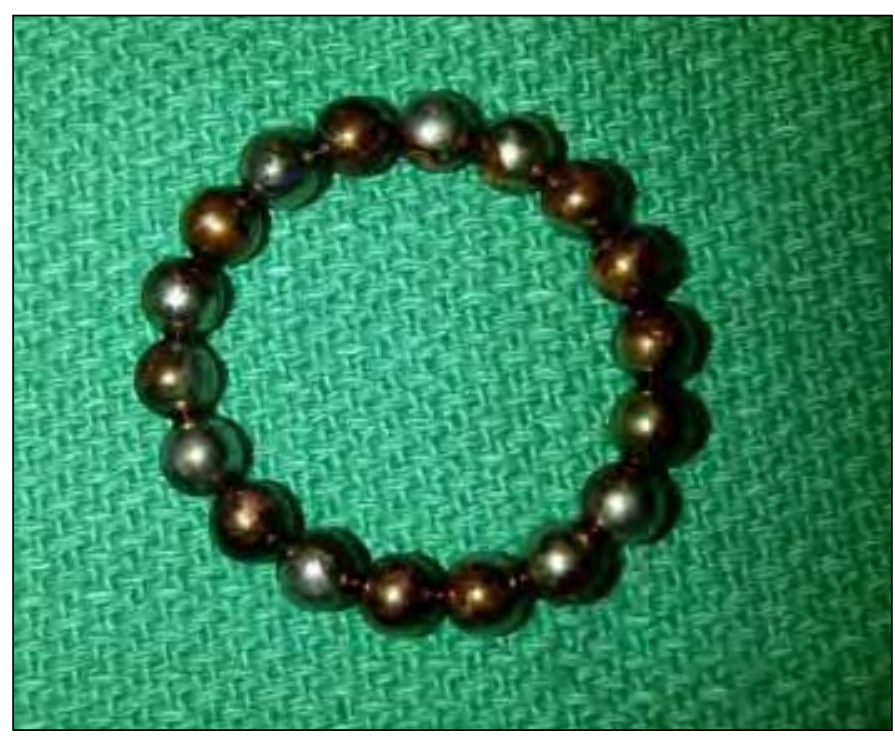

Fig. $\mathbf{2 b}$. Magnetic balls spontaneously arranged in ring post-extraction.

Further consideration should be given to the relative risks of any proposed extraction procedure. Both cystoscopic and open removal techniques carry their own inherent risks and benefits. The risks of rigid cystoscopy are generally minor, including urinary tract infection, hematuria and dysuria; rarer risks include tearing the urethra, a stricture of the urethra and the risks of general anesthesia. Likewise, open cystotomy carries the rare and minor risks which include risks of any surgery, namely bleeding, infection, thromboembolism and the risks of general anesthesia. Additionally, you need to consider the procedure-specific risks, such as urinary tract infection, hematuria and injury to other intraabdominal organs. Cystoscopy is less invasive and safer, but when cystoscopy is prolonged or technically difficult the risk of complication increases.

We suggest that when confronted with intravesical foreign bodies, operators consider open cystotomy as the preferred initial approach when the objects have a high propensity to alter their shape. In our example of multiple magnets, or in other cases such as insulated electrical wire, the pliability of the material created an occasion for open removal as a result of failed cystoscopic attempts. Interestingly, Graziottin and colleagues describe a similar case as ours, but they were successful with cystoscopic extraction. ${ }^{6}$ This came with a cost of significantly increased operative time and, as noted by the authors, increased the risk of urethral scarring in the future.

Bypassing a predictable cystoscopic failure may spare undue urethral injury. As was noted in Patient 1, the stenosis of the urethra suggested to the operators that the patient was not "insertion-naïve." It is likely that patients who present with accidentally retained foreign bodies in the bladder have attempted similar insertions before. ${ }^{5,6}$ Further urethral 
manipulation may contribute to delayed stenosis of the urethra. Additionally, when open retrieval is anticipated, foregoing endoscopy leads to removal of the foreign body sooner, reducing the amount of time between presentation and resolution.

As this was our third time seeing this particular foreign body in such a short time frame, it stands to reason that the manipulation of the urethra with these sort of beads may be a more common practice for certain people or groups. Therefore in a patient with foreign bodies of this nature, an attempt at endoscopic removal will no doubt be a fruitless endeavour; the patient should be taken directly for open removal.

\section{Conclusion}

Failure of cystoscopic methods for removal of intravesical foreign bodies may be predicted when an object's mechanical characteristics are considered. When foreseeable failure of endoscopic techniques can be anticipated, open removal should be the first-line approach.
Competing interests: None declared.

This paper has been peer-reviewed.

\section{References}

1. Rafique M. Intravesical foreign bodies: Review and current management strategies. Urol J 2008;5:223-31.

2. Moon SJ, Kim DH, Chung JH, et al. Unusual foreign bodies in the urinary bladder and urethra due to autoerotism. Int Neurourol J 2010;14:186-9. http://dx.doi.org/10.5213/ini.2010.14.3.186

3. Eradi B, Shenoy MU. Laparoscopic extraction of the awkward intravesical foreign body: A point of technique. Surg Laparosc Endosc Percutan Tech 2008;18:75-6. http://dx.doi.org/10.1097/ SLE.0b013e318157315f

4. Osca JM, Broseta E, Server $\mathrm{G}$, et al. Unusual foreign bodies in the urethra and bladder. $\mathrm{Br} J \mathrm{Urol}$ 1991;68:510-2. http://dx.doi.org/10.1111/i.1464-410X.1991.tb15395.x

5. Ayyildiz A, Gurdal $M$, Nuhoglu $B$, et al. A foreign body self-inserted via the urethra into the bladder: Pocket battery. Int Urol Nephrol 2003;35:251-2. htrp://dx.doi.org/10.1023/B:UROL.0000020290.56695.19

6. Graziottin TM, Soares DdFG, Da Ros CT, et al. Magnetic Spheres as Foreign Body into the Bladder. I Sex Med 2012 May 21. http://dx.doi.org/10.1111/j.1743-6109.2012.02772.x [Epub ahead of print]

Correspondence: Dr. Howard Evans, Division of Urology, University of Alberta, Suite 400 Hys Centre, 11010-101 St., Edmonton, AB T5H 4B9; hevans@aburologyinstitute.com 